\title{
COMPACTION OF METAL-CERAMIC POWDER MIXTURE. PART.1 (OVERVIEW)
}

\author{
Ileana Nicoleta Popescu ${ }^{1}$ And Ruxandra Vidu ${ }^{2,}$ \\ ${ }^{1}$ Valahia University of Targoviste, Faculty of Materials and Mechanics \\ Engineering, Campus, 13 Aleea Sinaia, 130004, Romania. \\ ${ }^{2}$ California Solar Energy Collaborative, University of California, Davis, One \\ Shields Ave, Davis, USA. \\ pinicoleta24@yahoo.com, " rvidu@ucdavis.edu
}

\begin{abstract}
The strength of green compact is influenced by the characteristics of the powders (apparent or tap density, particle size and shape, internal pores etc.), the processing parameters (applied force, pressing type, temperature) and testing conditions (strain rate etc.). For elucidation the phenomena that arise in the compaction of metallic and ceramic powders mixtures, in the first part of the paper, we present the mechanisms that influence the densification behaviour, the post-elastic effect, the compressibility of different (e.g. $\mathrm{Al}$ or $\mathrm{Cu}$ alloys $-\mathrm{SiC}_{\mathrm{Al}} \mathrm{O}_{3} / \mathrm{TiO} 2$ ) mixture powders and the particularities of each of them during the three stages of compaction.
\end{abstract}

Keywords: Densification mechanism, Stages of compaction, Compressibility, ElasticPlastic deformations, Metal-ceramic powders.

\section{Introduction}

The basic purpose of compaction is to obtain a green compact with sufficient strength to withstand further handling operations. Successful powder cold compaction is determined by the densification mechanisms and the behavior of the powder during compaction. The compressing of powder mixtures to obtain metal-ceramic composite parts can be done in different ways (with isostatic press, by rolling, extrusion) but compaction in dies using conventional hydraulic presses is the easiest method to obtain a green compact .For understanding the factors that determine a required strength, is necessary to investigate the densification mechanisms of mixture powders.

\section{Densification Mechanism. Elastic- Plastic Deformations of Powders}

According to the densification theories of the compaction powders proposed by Gethin, Sheppard, McShane, Fischmeister \& Artz [13], generally accepted, structural transformations at compaction operations are carried out in the following three stages (fig. 1) [4-10]:

StageI: Reorientation/
rearrangement and packing
particulate powders
Stage II: The increase of the
contact surface areas
between the particles by
elastic-plastic deformation
of the metallic particles and
the fragmentation of the
brittle ceramic particles

Stage III: Massive plastic deformation, porosity reduction

Figure 1. Structural transformations in three stages, during compaction operations according with Gethin \& Arin [9], Sheppard and McShane [10] , Fischmeister \& Artz [[11]; 
In the first stage of compaction, it takes place the decreasing the distance between the powder particles at the maximum level (form a closely packed mass) and the larger voids fill by reorienting, sliding, and interpenetrating the particles at very low pressures, according to Jones theory at around 0.05 -1 MPa [5]. The particles retain most of their original properties, although energy is dissipated due to inter-particle and particle to wall friction.

The second stage occurs at an intermediate pressure when the powder mass decreases continuously its volume. Due to the plastic deformation of the metal particles and the fragmentation of the hard/brittle - ceramic particles, the increase of the contact surfaces between the particles results.

The number of contacts increases as particle rearrangement and sliding occur. Cold welding and/or mechanical interlocking of the particles contribute towards the green strength of the compact. For brittle particles, the onset of plastic deformation can lead to fracture, giving way to fragmentation of the original particles and causing densification by repacking of the fragments.

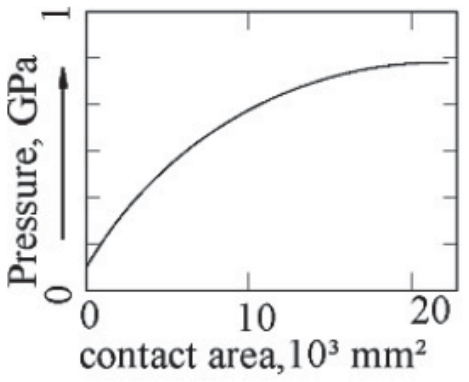

Figure 2. Variation of Contact area, Number of contacts and Porosity of spherical bronze particles with applied pressure [10].
Quantitatively, the contact surface area $S$ is directly proportional to the applied pressure $P$ to the mass of powders and also depends to the critical stress of plastic deformation or yield strength $\sigma_{c}$ of the material powder The correlation between the above mentioned parameters is

$$
P=\sigma_{c} S
$$

$\sigma_{c}$ is closely related to the hardness of powders. As the hardness of the metallic powder increases during the compaction (strengthening mechanisms), then the compression force must be chosen to be greater than the value calculated on the basis of Eq. (1). The contact area variation, the number of contacts and the porosity of the spherical bronze particles is shown in Fig. 2. According to Fig. 2, the number of contacts and the contact surface between the particles increases with the increase of the pressure, without decreasing the porosity $[11,12]$.

\section{Stress-Strain relations in densification mechanism}

According to the mixture rule, the composite yield strengths is the weighted average of the yield strengths of the components:

$$
\sigma_{c}=\sigma_{p} V_{p}+\left(1-V_{p}\right) \sigma_{m}
$$

where $\sigma_{c}, \sigma_{m}, \sigma_{p}$, represent the yield strengths of the composite, matrix or particles and the $V_{p}$ represent the volume (fraction) proportion of the particles. Due to the resistance of the material against deformation (external stress), the internal stresses in the particles increases. If the applied load is 
released before the deformation reaches a specific critical value, the particles will deform elastically.

Thus, when increasing the volume fractions $V_{c}$ of hard ceramic (e.g. SiC) particles in soft matrix (e.g. Al alloys), the yield strength of the composite increases and the contact surface areas of the mixtures at the same pressure will decrease. The deformation is reversible and the particles return to their original shape. Before this critical value, the stress is linearly proportional to the deformation [9] and is characterized by the elastic or Young's modulus (E, Fig. 3a). [10].

The second stage can be divided also into two major mechanisms: (i) the brittle ceramic particles are fractured at a certain stress value of the $\sigma_{f}$ (fracture strength). The brittle particles may fracture under stress, leading to mechanical interlocking (brittle behaviour (Fig. 3b); (ii) after reaching the critical stress $\sigma_{y}$, the ductile particles begin to deform plastically: (1) ductile behaviour with normal plastic flow (Fig. $3 \mathrm{c}_{1}$ ) or (2) they have ductile behaviour with strain-hardening (the material breaking eventually occurs at very high deformations, Fig. $3 c_{2}$.).

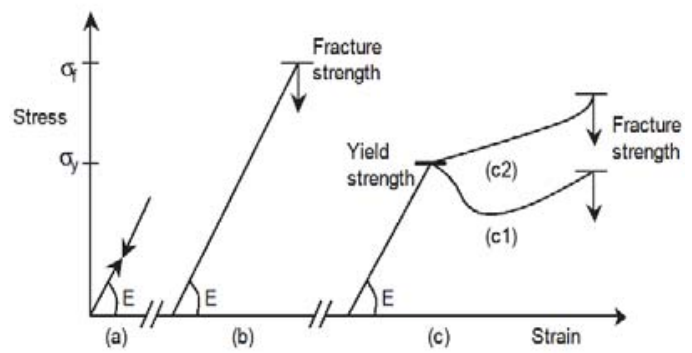

Figure 3. Macroscopic stress-strain relations: a) reversible elastic deformation; b) brittle behaviour; c) ductile behaviour (c1. normal plastic flow; c2. strain-hardening) [10].

The way when powdered materials behave ductile, fragile or ductile-fragile under the action of compressive forces depends on the material and its physical conditions.
In order to model the cold compression behaviour of metal-ceramic powder mixtures (a mixture of hard and soft particles) several simplifications have been made, such as that the mixtures are homogeneous, the contact forces between the particles are equal in the same direction. The particles have a spherical shape of the same or different dimensions [13] or dimensions or take into account the frictional forces between the particles.

For elucidation of the phenomena that arise in the compaction of metallic and ceramic powders mixtures, Lange et al. [14] have developed a model of hard and soft spheres of different dimensions. The authors suggest two main mechanisms for explaining cold compression behaviour:

(1) the densification of each composite mixture in comparison with densification of exclusively soft powders (the powders for soft, metallic matrix). In this case, the densification can be inhibited by the presence of hard particles, which is confirmed by the fact that the soft particles of metallic material suffer an additional deformation due to the partial filling of the areas around the hard particles;

(2) the loads are not entirely transmitted to the soft particles due to the formation of a continuous network of hard particles that take up some of the load.

Martin and Bouvard [13] have also come to the same conclusion by modelling the compression behaviour of hard and soft powder mixtures by DEM method, namely that the hard particles carry a large part of the pressing load as well as a part of the deformation of the soft particles. Bouvard and Lange [15] proposed an analytical approach combined with a numerical simulation of the infiltration of the hard particles between the soft particles according to the size and fraction of the hard and soft particles and confirmed that according to the experimental investigations densification is much more difficult for mixtures of metallic and ceramic 
powders over metallic ones [16]. The degree of densification is influenced by the size, shape, surface state and particle distribution. Cazotti and others [7] have shown that the large particles are deformed more easily than thick ones, and densification is greater in this case, ie at much lower pressures than fine powders.

C. Ghita and I.N. Popescu [6] respectively S.Sivasankaran et. al. [17] have been demonstrated by them experimental researches that by adding the hard and fragile powders in the soft aluminium alloy powder mixtures, the compressibility decreases, this decreasing is in accordance with the experimental compressibility curves (Fig.4 and 5).

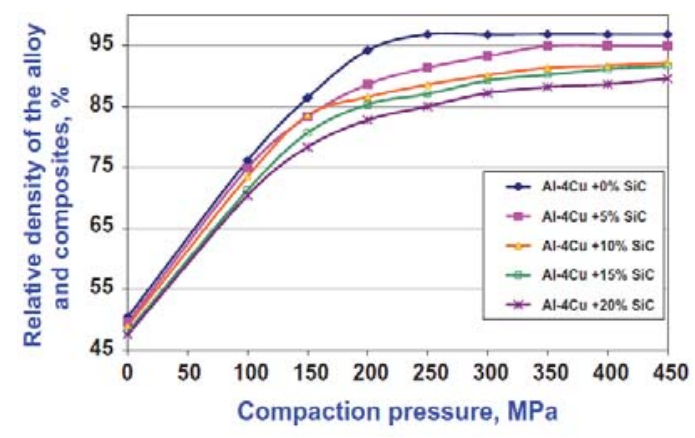

Figure 4. The densification curves for $\mathrm{Al} 4 \mathrm{Cu} / \mathrm{SiCp}$ composites [6].

The effect of the nanocrystallite matrix particle size reduction due to incorporation of nano-sized titania considerably reduced the relative density at low compaction pressure ( $<250 \mathrm{MPa})$. This decreased relative density with the increase of nano-sized titania content in the nanocrystallite matrix in the particle rearrangement mechanism domain was due to work hardening effect. On the other hand at high compaction pressure the rate of decreased relative density in the plastic deformation domain was lower than particle rearrangement mechanism due to drastic powder morphology reduction. Also, this decreased relative density was also due to increasing of stress shielding effect by titania addition [17].

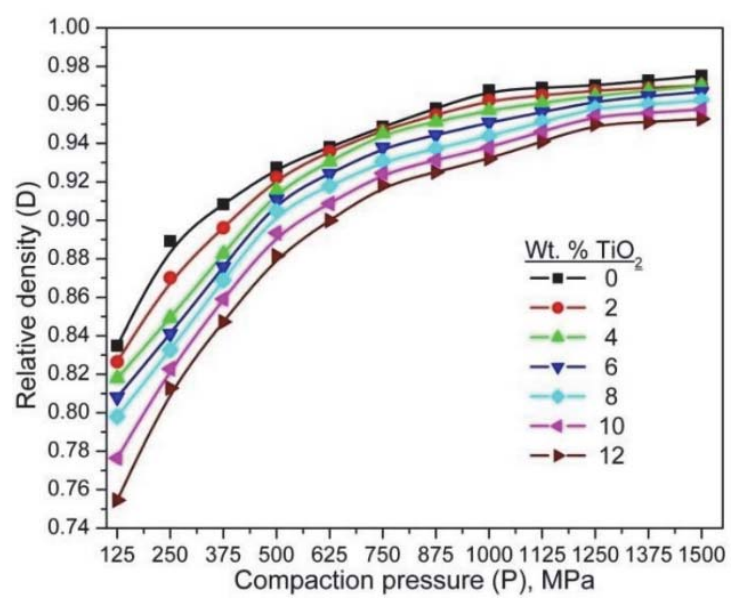

(i)Particle rearrangement (PR)

(ii) Plastic deformation (PD)
PR<375 Mpa

Both-375 to $1000 \mathrm{Mpa}$ PD $>1000 \mathrm{Mpa}$
Figure 5. Compressibility curves of AA6061$\mathrm{x}$ wt. $\% \mathrm{TiO}_{2}(\mathrm{x}=0,2,4,6,8,10$ and 12) nanocomposite powders as a function of compaction pressure at Various $\mathrm{TiO}_{2}$ [17].

Also, Cazzoti et.al. [7] confirm that the presence of the $\mathrm{Al}_{2} \mathrm{O}_{3}$ reinforcing particles with larger size in aluminium matrix composite produces an increase in the compressibility of the powder when $5 \%$ and $10 \%$ by volume of reinforcement is added. But the addition of $15 \% \quad \mathrm{Al}_{2} \mathrm{O}_{3}$ of this reinforcement causes the opposite effect, that is, it decreases the compressibility of the powders [7] (Fig. 6).

Roberts and Rowe [18, 19] demonstrated that materials possess a critical particle diameter at which the densification mechanism turns from fragile to ductile and the particle size decreases (Fig. 7).

The stress necessary to cause particle fracture increases when the particle size decreases, whereas the stress causing plastic deformation of a material is independent of the particle size. 

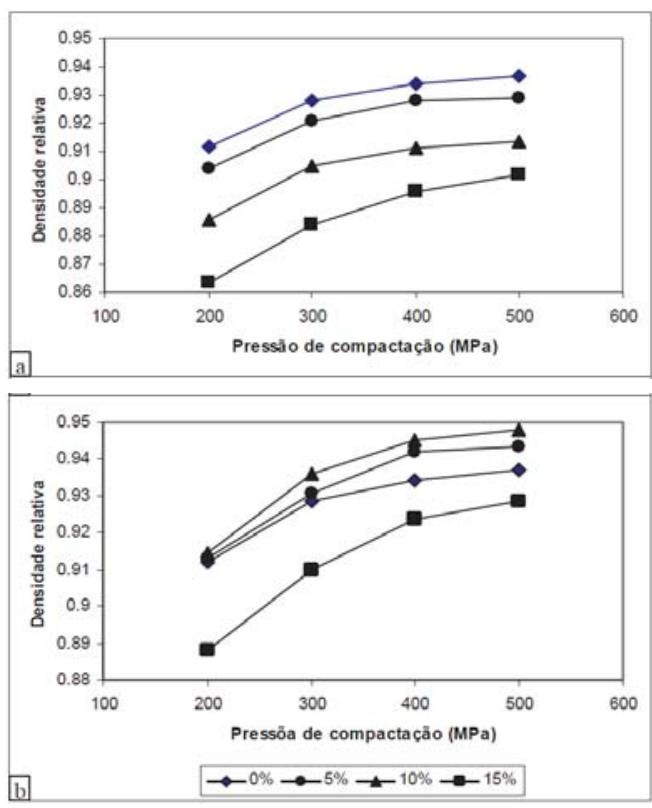

Figure 6. Compressibility curves of ALUMIX $123 \circledR$ aluminum powder ( $0 \%$ reinforcement) and reinforced composites with different amounts of $\mathrm{Al} 2 \mathrm{O} 3$ powder of smaller size (a) and larger size (b).

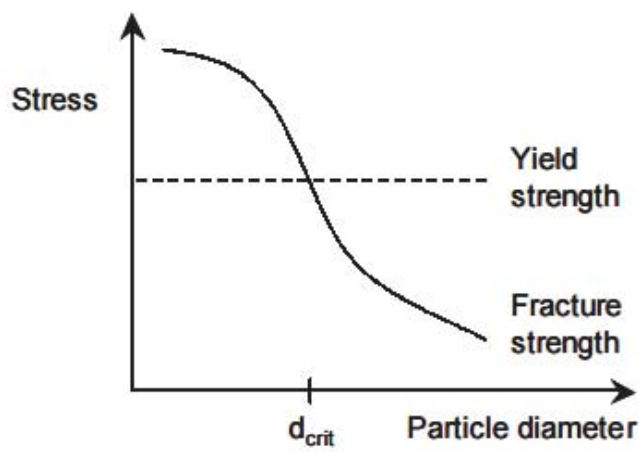

Figure 7. Schematic representation of the particle size effect on yield strength or fracture strength $[18,19]$.

When the fracture stress reaches the level of the yield strength, particles with diameters lower than the critical diameter will yield instead of fracturing. Achieving a dense packing of particulate powders involves a large particle mobility (powders fluidity) characterized quantitatively by the apparent and relative density of the powders, the porosity of the material and the flow rate of the powder.

Powders with irregularly shaped particles with laced edges and rough surface flow more heavily than spherical shapes due to friction between the particles and thus will have a lower packing degree and a less compaction behaviour. Instead, particles with spherical particles (in the case of a mixture of unreinforced aluminium powders or low reinforcement particles with $\mathrm{SiC}$ particles [20]) and a smooth surface, perform better when pressed, giving more compact powders by a better interlocking of particles, sliding and rotation of them.

Additionally, the packaging is more regular, resulting in a uniformly distributed porosity when powders are compressed. Better packaging has been shown to be in the case of different grains - for example a mixture of two spherical particle fractions, a fraction of $1 \mathrm{~mm}$ in diameter and the other one with a diameter of $0.001 \mathrm{~mm}$ - when fine particles can hypothetically occupy the largest voids. In this ideal case, the compaction increasing to about $80 \%$.

For this reason, the actual porosity of the experimental powders is higher than that calculated on the basis of systematic packaging models. [21]

Similarly, Zavaliangos and Laptev [22] demonstrated the importance of the relative particle arrangement during compression. These authors have shown that cyclical pressures greatly enhance composite material consolidation, leading to better packaging. Another important factor of the density of the respective densification is the ratio of the particle sizes in the mixture: the high ratio of the particles increases the degree of compaction but in a proportion that does not cause separation of the large particles by the fine particles. If there is a large amount of fine powder in relation to the thick (coarse) ones, the porosity will increase [20]. With further increase of applied pressure, the number of 
contacts increases and the density of the compacted bulk tending towards to the true densities of the component ingredients, leading to porosity decreasing (stage III) [4-12].

This is explained by packaging repackaging of metallic and ceramic particles at higher pressures. In the course of compaction not only plastic deformation of the particles occurs, but in some particles the deformation is elastic. When removing the green compacts from the die, these elastic deformations (compression) disappear and a sudden expansion of the workpiece volume occurs (the post-elastic effect appears) [23].

\section{The post-elastic effect}

During and after the removal of the applied load, a compressed powder generally shows an elastic recovery of its volume as an effect of stress relaxation. In case of a permanent deformation, the volume expansion will be small, whereas large compact powder relaxations are caused by an important elastic component The compact powder relaxation is commonly expressed by a change in compact height, volume or porosity [23].

The green compact expansion expressed as porosity expansion was found to be independent of the applied load. The green compact relaxation is influenced by the compaction speed [24] and particle size. The post-elastic effect of hard and fragile green compact powder mixtures is higher than that obtained from soft and plastic materials. This is explained by the fact that, at the same compression pressure, the strength of the hard particle compact is lower than the soft particles, thus increasing the role of the elastic deformations compared to the plastic ones[20, 25].

\section{Conclusions}

The high powder compression capacity (best densification) is given by:

(a) smooth, regular particle surface; (b) different particle size grains, thus choosing the fine particles to fill the voids created by the coarse particles;

(c) the most part of the pressing die volume is occupied by coarse particles; the difference in size between the fine particles and the thick ones is very high;

(d) the quantity of fine particles is not very large in relation to the thick ones in order not to separate them;

(e) lower hardness (high plasticity) of the particles or the existence in the smallest quantities of composites of hard ceramic particles; (f) particles with as little oxide as possible (the oxygen content of the powder must not exceed $1.5 \%$ ).

\section{References}

[1] D. Gethin, A.K. Arin, D.V. Tran, R.W Lewis, Powder Metallurgy, 37(1) (1994). 42.

[2] T. Sheppard, H.B.McShane, Powder Metallurgy, 23 (3).(1980).120.

[3] F. Fischmeister, HE. Arzt, Powder Metallurgy, 26(2) (1983). 82.

[4] R.M.German, Particle Packing Characteristics, MPIF, Princeton, NJ, 1989.

[5] W.D. Jones, Fundamental Principles of Powder Metallurgy, Edward Arnold Publishers, London, 1960.

[6] C. Ghiţă, I.N. Popescu, Comp Mater Sci, 64 (2012). 136.

[7] C. Cazotti, J.L.A. Oliveira, J.B. Fogagnolo, 17o Proceedings of CBECIMat, $15-19$ de November, Foz do Iguaçu, PR, Brasil (2006) 7452 .

[8] R. Panelli , F Ambrózio Filho, Powder Metallurgy, 41(2).(1998).131.

[9] C. Ghiţă, N. Pop, I.N. Popescu, Comp Mater Sci, 64 (2012).52.

[10]van Veen, B. Compaction of powder blends: effect of pores, particles and percolation on tablet strength, Groningen (2003).

[11]H.F. Fischmeister, E. Arzt, L.R.Olsson, Powder Metallurgy 21 (4) (1978).179.
[12]R.M.German,
Particle
Packing

Characteristics, (MPIF), Princeton, NJ, 1989. 
[13]E. Arzt, Acta metall. mater., 30 (1982).1883.

[14]F.F. Lange, L. Atteraas, F. Zok, J.R. Porter. Acta Metall. Mater. 39 (1991).209.

[15] D Bouvard, FF. Lange, Acta Metall Mater 39(12)( 1991). 3083.

[16] C.D. Turner, M.F. Ashby, , Acta Mater. 44 (1996). 4521.

[17]S. Sivasankaran, K. Sivaprasad, R. Narayanasamy, Vijay Kumar Iyer, Powder Technology 209 (2011) 124.

[18] R.J.Roberts, R.C . Rowe, Chem. Eng. Sci., 42(4) (1987) 903.

[19] R.J.Roberts, R.C.Rowe, Int. J. Pharm., 36 (1987) 205.

[20] I.N. Popescu, S. Zamfir F. V.Anghelina, C.O. Rusănescu, Proceedings of the 2nd Int. Conference on MEQAPS, Constantza, Sep 03,05(2010) .200.

[21] S. Joel Hirshborn, Introduction to Powder Metallurgy, 1969.

[22] A. Zavaliangos, A.Laptev, ACTA MATER ;48(10)( 2000).2565.

[23] K .Van der Voort Maarschalk, K. Zuurman, et.al, Int. J. Pharm., 140 (1996).185.

[24] K .Van der Voort Maarschalk, H.Vromans, et.al. , Eur. J. Pharm. Biopharm., 42 (1996). 49.

[25] I.N. Popescu, Researches on development of new composite materials with Al and Al alloys matrix, Ph.D. Thesis, Bucharest, 2006. 